\title{
BEAM TEST OF COMPACT SR RING "AURORA-2S" FOR X-RAY LITHOGRAPHY
}

\author{
D. Amano, T. Hori, $\underline{\text { H. Miyade }}$, H. Murata, T. Takayama \\ Research \& Development Center, Sumitomo Heavy Industries, Ltd. \\ 2-1-1 Yato-cho, Tanashi-city, Tokyo 188-8585 Japan
}

\begin{abstract}
From January 1998, we started the beam test of "AURORA-2S" (A2S) with the same method as used for "AURORA-2D" (A2D) and were able to accumulate $0.7 \mathrm{GeV}$-electron beam in a comparatively short period. In the mean time, though, we found some problems while injection which were serious not for A2D but for A2S. In September 1998, however, we have succeeded in managing such the problems and obtained the following results so far; more than $1 \mathrm{~A}$ of injected current with maximum increasing rate of $640 \mathrm{~mA} / \mathrm{min}$, and stored current exceeding the specification $500 \mathrm{~mA}$ with typical acceleration efficiency over $80 \%$. Since then the A2S has been operated without any serious troubles, in February 1999 the integrated current exceeded 110A.H and the beam lifetime reached $150 \mathrm{~min}$ at $500 \mathrm{~mA}$. Moreover the acceleration efficiency increased to $97 \%$.
\end{abstract}

\section{INTRODUCTION}

"AURORA-2" (A2) is a new type compact SR source. The outstanding feature lies in normal conducting bending magnets excited up to 2.7 Tesla, therefore operation and maintenance of the ring are simplified in the results compared with the superconducting's[1].

One of two types of A2 is "AURORA-2D" (A2D) for the scientific research. A2D has two long straight sections where insertion devices can be installed. A2D is at Hiroshima University, named HiSOR, with two undulators and has been routinely operated since 1997[2].

The other is "AURORA-2S" (A2S), which is optimised for the industrial use, mainly X-ray lithography. For A2S the straight sections are shortened and the whole size becomes as small as possible. A2S is designed as the size of the electron beam keeps uniformity in the bending magnet, therefore it can provide same light through each port. Furthermore lead and polyethylene surround the whole machine for radiation shielding so that the wall of the machine room can be thinned.

Parameters of A2S are summarised in Table 1 and the schematic view is shown in Fig. 1.
Table 1:Parameters of AURORA-2S

\begin{tabular}{|c|c|c|}
\hline \multirow{2}{*}{$\begin{array}{c}\text { Energy: Storage } \\
\text { Injection }\end{array}$} & 0.7 & $\mathrm{GeV}$ \\
\hline & 0.15 & $\mathrm{GeV}$ \\
\hline Circumference & 10.97 & $\mathrm{~m}$ \\
\hline Harmonic number & 7 & \\
\hline RF frequency & 191.2 & $\mathrm{MHz}$ \\
\hline Energy Loss & 24.42 & $\mathrm{keV} /$ turn \\
\hline $\begin{array}{l}\text { Tune: } \\
\begin{array}{c}\text { horizontal } \\
\text { vertical }\end{array}\end{array}$ & $\begin{array}{l}1.46 \\
0.73\end{array}$ & \\
\hline Natural emittance & 527.6 & $\pi$ nm.rad \\
\hline Radiation damping: & & \\
\hline horizontal & 2.13 & msec \\
\hline vertical & 2.10 & msec \\
\hline longitudinal & 1.04 & msec \\
\hline Touschek life time & 6.6 & $\begin{array}{l}\text { hours } \\
\text { (at 1A) }\end{array}$ \\
\hline
\end{tabular}

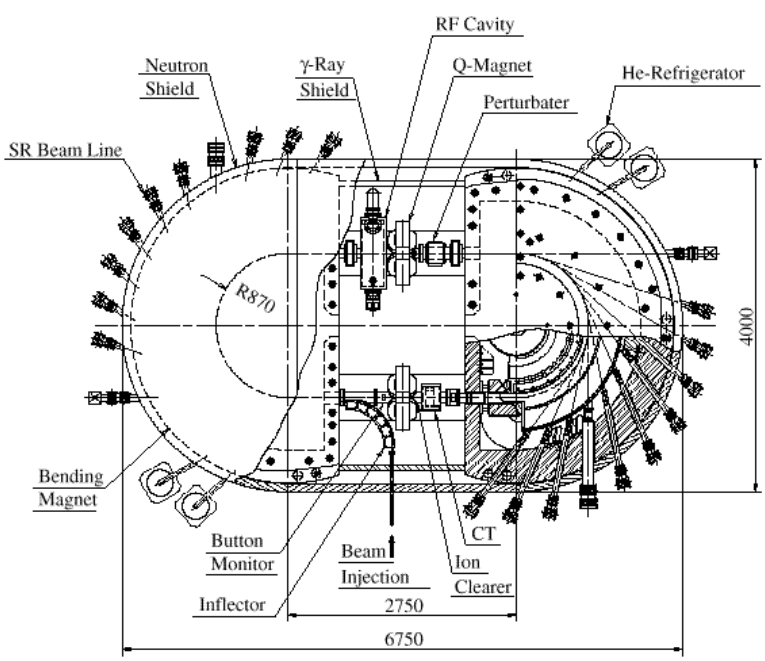

Figure 1: Schematic view of AURORA-2S

\section{BEAM TEST OF A2S}

\subsection{Results and Problems of the First Beam Test}

First we constructed A2D in our laboratory and made a performance test in 1997. After obtaining satisfactory results[3],[4] the A2D was reassembled to A2S.

The first beam test was started from January 1998 with the same method of beam injection, acceleration, and

\footnotetext{
*Email: Hrk_Miyade@ @hi.co.jp
} 
accumulation as used for A2D[3], and then we succeeded in accelerating the electron beam up to $0.7 \mathrm{GeV}$ in about a week. After then we optimised various parameters and finally obtained the following results[5].

- Maximum injection current: $340 \mathrm{~mA}$

- Maximum stored current : $110 \mathrm{~mA}$

- Acceleration efficiency : $70 \%$

The exciting rate of the main magnetic field was $6 \mathrm{~A} / \mathrm{s}$ in constant and the time during excitation was about $2 \mathrm{~min}$.

After that, however, we found some problems while injection which were serious not for A2D but for A2S and we could not get better performance. The problems were as follows;

- The beam could not be injected at the designed operation point $(v \mathrm{x}=1.46, \mathrm{vy}=0.73)$, but only at a different point $(v \mathrm{x} \sim 1.3, v \mathrm{y} \sim 0.8)$.

- The instability might occur. Changing in position of the tuners in the cavity could diminish it a little. But it was powerless in the rapid varying situation such as in acceleration.

- $\quad$ Radiation from the light port was abnormally intense. It might be suggested that much of the electron beam were lost at the ion clearer in the bending magnet.

\subsection{Counterplans to the Problems}

After that we found an effect of the perturbater (PTB), which is a kicker magnet for injection, with a simulation; such that during the excitation of PTB the circumference became too short in the operation at the designed point, then the difference between the revolution time and the $\mathrm{RF}$ cycle grew more and more, at last it reached 150 degrees in RF phase.

To correct the difference we tried the method that the frequency modulation (FM) was piled up on the RF in a series of pulses simultaneously with the excitation of PTB. The shape of the pulse was same as the excitation pattern of PTB, that was a half-sine wave with $1 \mu \mathrm{sec}$ width. The width of FM was about $500 \mathrm{kHz}$ at the peak.

During the first beam test, in the cavity there were two tuners, a remote-controlled and a manual-controlled. After the test we made one more remote-controlled tuner, therefore we had been able to control two tuners remotely and one manually. Moreover we made the speed of motors of the tuners faster. With this we had been able to operate the machine in wider range and more quickly.

For the problem of the intense radiation we removed the ion clearer. In the vacuum chamber whose inner half height was $15 \mathrm{~mm}$, the ion clearer was installed with a distance of $4 \mathrm{~mm}$ from the bottom of the chamber. Removing it, the aperture was extended from $26 \mathrm{~mm}$ to $30 \mathrm{~mm}$.

As soon as we started the second beam test from September 1998, we found the effect of the reconstruction; such that the beam could be injected near the designed operation point and the injection speed increased extremely. Then we succeeded in accumulation of the specification $500 \mathrm{~mA}$.

Fig. 2 shows the behaviour of the beam when the FM is on (left) or off (right). They were observed with the streak camera. In each figure the ordinate means the time with $120 \mu \mathrm{sec}$ full scale and the abscissa means the horizontal deviation of the beam with an arbitrary unit. The steep peak on the upper side represents the moment when the PTB was excited. In FM-OFF, a large oscillation, which may be a synchrotron oscillation, occurs according to the excitation of PTB. On the other hand in FM-ON the oscillation is inhibited.
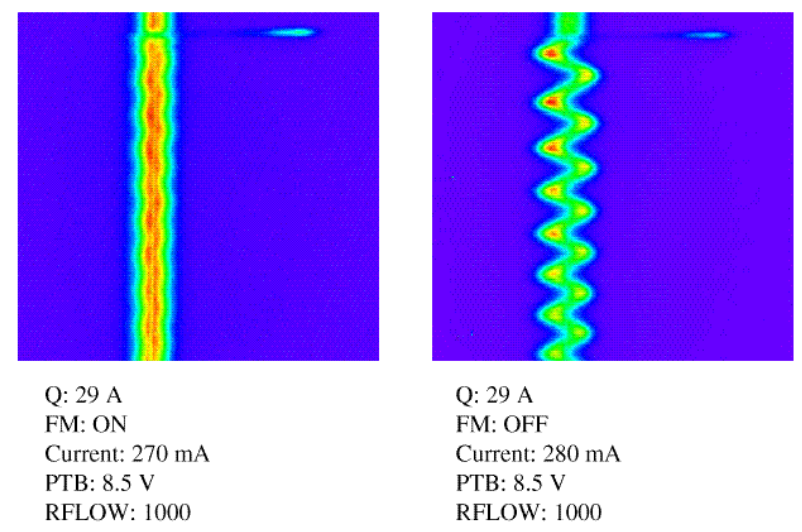

Figure 2: Effect of frequency modulation on RF

In the second beam test the following results were obtained;

- Maximum injection current: $1020 \mathrm{~mA}$

- Maximum stored current : $>500 \mathrm{~mA}$

- Maximum injection speed : $640 \mathrm{~mA} / \mathrm{min}$

- Acceleration efficiency : $>80 \%$

\subsection{Routine Operation}

In November 1998 we installed a new cavity and a Landau cavity. They will be mentioned in the next section. In this subsection we will mention the third beam test.

The third beam test started from February 1999. The main purposes were ageing the vacuum chamber and lengthening the lifetime. To make an ageing efficiently the integrated current was needed as much as possible in a short period. So we needed to operate the machine without stopping in the test period and therefore some of members in our laboratory, they were not necessarily experts on the operation, were needed to operate it. From November 1998 to February 1999 we made an effort to simplify the operation for the amateur operators but not to increase the stored current nor to research the effect of the new and the Landau cavities. 
The test had been done for two weeks ( 272 hours) with stored current of $500 \mathrm{~mA}$ routinely. There were no devices damaged or troubled during the test, so we could make sure the high reliability of A2S. Finally the integrated current exceeded 110A.H (including 25A.H before the test) and the lifetime ${ }^{\#}$ reached $150 \mathrm{~min}$ at $500 \mathrm{~mA}$ and $240 \mathrm{~min}$ at $300 \mathrm{~mA}$ (see Fig.3). Moreover the injection efficiency increased to more than $97 \%$. However these were not satisfactory results, because the lifetime was shorter than we expected. This means the ageing was not enough. In fact the vacuum pressure in the main cavity was $1.0 \times 10^{-6} \mathrm{~Pa}$ at $500 \mathrm{~mA}$ and was an order of magnitude higher than our expectation. To reach the enough vacuum pressure and the satisfactorily long lifetime, we need more several hundreds A.H or the pumping system should be reinforced.

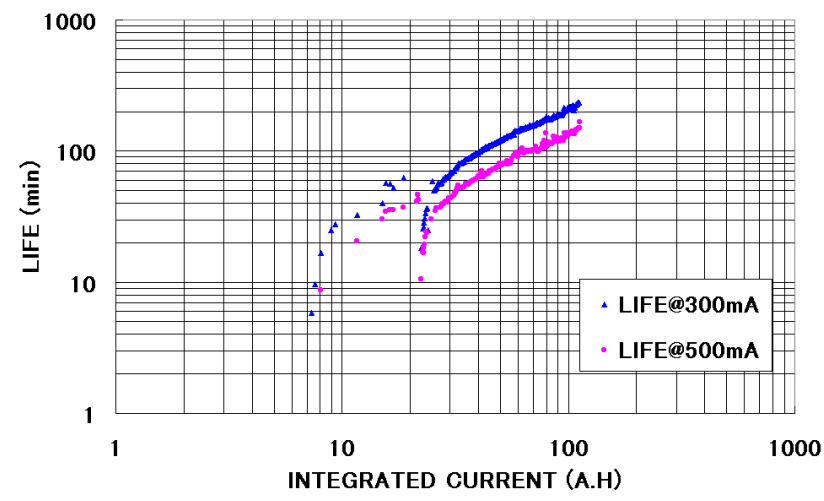

Figure 3: Integrated current and lifetime

\section{NEXT STEP}

With the above test the performance of A2S has reached almost full specification. But there still exist some problems. One of them is the existence of the instability. We can still observe the synchrotron oscillation occurs even in storing beam with the energy of $0.7 \mathrm{GeV}$. Even under the instability we could accumulate the beam up to $500 \mathrm{~mA}$ and the light from the ring had no problems for use, however it might disturb trying to accumulate the beam more than $500 \mathrm{~mA}$.

The instability is caused by a higher order mode electromagnetic field (HOM) in the cavity. So we designed and constructed a new cavity, which had less HOMs than the old cavity had (see Fig. 4). Moreover also a Landau cavity whose fundamental frequency is three times higher than the main cavity's was constructed. The new cavity and the Landau cavity have already been installed in the ring in November 1998.

\#The lifetime ' $\tau$ ' is defined as follows;

$$
\tau=-I /(d I / d t)
$$

Our next step is to diminish the instability with the new cavity and the Landau cavity, and to achieve the upgraded goal, $1000 \mathrm{~mA}$ of stored current at $0.7 \mathrm{GeV}$.


Figure 4: Characteristics in frequency with the old (upper) and the new (lower) cavities

\section{REFERENCES}

[1] T. Hori, et al., "AURORA-2: Compact Advanced SR Ring as an Xray Source", 4th Int'l Conf. On SR sources \& 2nd Asian Forum on SR, Kyongju, Korea (1995) pp.148-158

[2] K. Yoshida, et al., "Commissioning of a Compact Synchrotron Radiation Source at Hiroshima University”, APAC'98, KEK, Tsukuba, Japan, March 23-27 1998

[3] T. Hori, "Present Status of AURORA-2D", 11th Symposium on Accel. Sci. and Tech., Harima, Japan (1997) pp.534-536

[4] T. Hori, et al., "Operation Results of Compact SR ring AURORA2D with 7Tsla Wiggler”, EPAC'98, Stockholm, Sweden (1998) pp.581-583

[5] H. Miyade, et al., "Initial Commissioning of Dedicated SR Ring “AURORA-2S" for X-ray Lithography", Epac'98, Stockholm, Sweden (1998) pp.2413-2415 